\title{
THE DEUTERIUM ABUNDANCE IN THE LOCAL INTERSTELLAR MEDIUM
}

\author{
JEFFREY L. LINSKY 1 \\ Joint Institute for Laboratory Astrophysics \\ NIST and University of Colorado \\ Boulder, Colorado 80309-0440 USA
}

\begin{abstract}
Accurate measurements of the interstellar deuterium/hydrogen ratio along different lines of sight are needed to test predictions of contemporary cosmologies. I describe our GHRS observations of the Lyman- $\alpha, 2600 \AA$ and $2800 \AA$ spectral regions for the spectroscopic binary system Capella, obtained with $3.57 \mathrm{~km} \mathrm{~s}^{-1}$ resolution and high signal/noise. We are now analyzing these data to infer the column densities of H I, D I, Mg II, and Fe II along this 13 pc line of sight.
\end{abstract}

\section{The Importance of Accurate Measurements of the D/H Ratio}

One of the major scientific objectives of the HST is to obtain data that will test and refine models of the early evolution of the universe. Observations with Hubble can address this question in three ways: measure the Hubble expansion parameter $\mathrm{H}_{\circ}$, estimate the baryon density of the universe and the amount of "dark" matter, and estimate the primordial light element abundances. Although accurate measurements of the primordial D, ${ }^{3} \mathrm{He},{ }^{4} \mathrm{He}$, and ${ }^{7} \mathrm{Li}$ abundances constrain early cosmologies, the $\mathrm{D} / \mathrm{H}$ ratio may be the most useful constraint, since the relationship of $\mathrm{D} / \mathrm{H}$ to the present mean density of the universe, and thus the closure parameter $\Omega$, is monotonic and is steepest for the light elements.

\section{Different Techniques for Measuring $\mathrm{D} / \mathrm{H}$}

The deuterium abundance in a largely neutral gas can be estimated from the strength of lines in deuterated molecules like HD, the 92-cm ground state hyperfine transition of atomic D, and the atomic deuterium Lyman lines. Chemical fractionation processes in cold interstellar clouds can lead to very unreliable $\mathrm{D} / \mathrm{H}$ ratios from measurements of deuterated molecules, and the $92-\mathrm{cm}$ line is exceedingly weak, but the third technique holds promise. The deuterium Lyman- $\alpha$ line lies at $-80 \mathrm{~km} \mathrm{~s}^{-1}$ $(-0.32 \AA)$ from the corresponding hydrogen line, and the higher members of the deuterium Lyman series are displaced by similar amounts relative to hydrogen.

The interstellar deuterium Lyman- $\alpha$ line may be detected in absorption against the continuum of a hot star or the Lyman- $\alpha$ emission line of a late-type star, provided that the deuterium line has sufficient opacity $\left(\tau_{D}>0.01\right)$ and the hydrogen line is

1Staff Member, Quantum Physics Division, National Institute of Standard and Technology 
not so opaque $\left(\log N_{H} \leq 18.5\right)$ that it obliterates the neighboring deuterium line. This requirement severely limits the number of available background sources to the nearby late-type stars, a few hot white dwarfs, and the very few O-type stars with exceptionally low column densities. One may expand the number and lengths of the useful lines of sight by obtaining high-resolution spectra of the higher members of the Lyman series. This was done for a few bright O-type stars by the Copernicus satellite (see Bruhweiler \& Vidal-Madjar 1987) and will be one of the main objectives of the FUSE satellite now in Phase B development. Extension of this program to distant regions of our Galaxy and beyond is needed to study matter where the D/H ratio may be close to its primordial value. For lines of sight in the local interstellar medium (LISM), one expects that the measured $\mathrm{D} / \mathrm{H}$ value will be lower than its primordial value due to deuterium burning in the cores of stars and the addition of this material to the interstellar medium by stellar winds and supernovae explosions. Thus measurements of $\mathrm{D} / \mathrm{H}$ in the LISM provide lower limits to the primordial value.

\section{Previous Studies of D/H Toward Nearby Cool Stars}

Copernicus and IUE have obtained spectra of the Lyman- $\alpha$ line for seven nearby late-type stars with derived $\mathrm{D} / \mathrm{H}$ ratios in the range $0.1-10 \times 10^{-5}$ (Murthy et al. 1990). The very uncertain values in $\mathrm{D} / \mathrm{H}$ derived from these spectra are due to the low signal/noise in the data and to the inadequate spectral resolution $\left(30 \mathrm{~km} \mathrm{~s}^{-1}\right.$ for IUE and $15 \mathrm{~km} \mathrm{~s}^{-1}$ for Copernicus). Since the deuterium line is not spectrally resolved, one must use curve-of-growth techniques. This leads to very uncertain deuterium column densities because the deuterium line is typically on the flat part of the curve of growth and there may be multiple velocity components. Even the hydrogen column density cannot be accurately estimated, since the line is not completely on the damping part of the curve of growth.

\section{Measuring $\mathrm{D} / \mathrm{H}$ with the GHRS}

Before the loss of Side 1, the GHRS provided ideal properties for measuring D/H using the Lyman- $\alpha$ line. The high spectral resolution of the Echelle-A grating (3.57 $\mathrm{km} \mathrm{s}^{-1}$ ) was sufficient to resolve the deuterium line, which is about $13 \mathrm{~km} \mathrm{~s}^{-1}$ wide at half intensity, and to identify individual velocity components separated by more than $\sim 2 \mathrm{~km} \mathrm{~s}^{-1}$. For brighter stars like Capella, signal/noise $\approx 100$ could be achieved with the FP-Split observing strategy, and the GHRS has a very accurate velocity scale. The small aperture $(0.25 \operatorname{arcsec} \times 0.25 \mathrm{arcsec})$ reduces the geocoronal Lyman$\alpha$ contamination to a negligible amount and provides a good instrumental profile with low scattered light. The price for obtaining these benefits is long exposure times (see Table 1). The spherical aberration in the primary mirror does not compromise data quality, but it increases the exposure time by a factor of 5 , thereby limiting the number of stars that can be observed. COSTAR will not increase the spectral resolution and will not restore the predicted throughput without aberration, because of reflection losses at two optical surfaces. The recovery of GHRS Side 1 capability (including Echelle-A) would greatly strengthen spectroscopy with Hubble, but even moderate resolution spectroscopy with Side 2 can increase our understanding of the $\mathrm{D} / \mathrm{H}$ ratio as a test of cosmological theories. 


\section{GHRS Observations of Capella}

Capella ( $\alpha$ Aurigae) is a 104-day period spectroscopic binary system consisting of a G9 III star and a rapidly-rotating G0 III star which, as shown in IUE spectra, dominates the ultraviolet emission line spectrum of the system (Ayres \& Linsky 1980). We selected Capella as the first target in what we anticipated would be a large observing program, because the integrated flux in its Lyman- $\alpha$ emission line is the largest of any late-type star and is thus a good background source against which to measure the hydrogen and deuterium interstellar absorption. Table 1 summarizes our observations of Capella on 1991 April 15 near phase 0.28 when the radial velocity separation of the two stars was $53.5 \mathrm{~km} \mathrm{~s}^{-1}$.

Table 1. Summary of GHRS Observations

\begin{tabular}{|cccccc|}
\hline Grating & Aperture & $\begin{array}{c}\text { Spectral } \\
\text { Range }\end{array}$ & $\begin{array}{c}\text { Spectral } \\
\text { Resolution } \\
\lambda / \Delta \lambda\end{array}$ & $\begin{array}{c}\text { Exposure } \\
\text { Time } \\
\text { (sec) }\end{array}$ & $\begin{array}{c}\text { Spectral } \\
\text { Features }\end{array}$ \\
\hline EA-46 & SSA & $1211-1217 \AA$ & 90,000 & 3917 & HI 1216 $\AA$, DI $1215 \AA$ \\
EB-22 & SSA & $2594-2606 \AA$ & 90,000 & 707 & FeII 2599 \\
EB-20 & SSA & $2793-2807 \AA$ & 90,000 & 707 & MgII $2796,2803 \AA$ \\
\hline
\end{tabular}

The Ech-A spectrum of the Lyman- $\alpha$ region (Figure 1) shows the broad stellar Lyman- $\alpha$ emission line and the interstellar hydrogen Lyman- $\alpha$ absorption feature and a narrow interstellar feature due to deuterium Lyman- $\alpha$ centered at $-0.32 \AA$ relative to the hydrogen absorption line. The deuterium line has been seen in Copernicus and IUE spectra of Capella (e.g. Murthy et al. 1990) and other stars, but this spectrum is the first in which the line has been spectrally resolved. The small amount of instrumental scattered light can be measured from the minimum flux seen in the saturated interstellar core of the hydrogen absorption line. The central depth of the deuterium feature is a measure of its optical depth, and the shape of the hydrogen absorption feature can be used to measure its opacity.

Figure 2 compares the interstellar absorption lines of $\mathrm{Mg}$ II h, Fe II $2600 \AA$, and deuterium on a common wavelength scale. Since the D and Fe ions differ by a factor of 28 in mass, the different line widths provide a means for separating thermal from turbulent broadening. There appears to be only one velocity component in this line of sight. When we better understand the instrumental properties of the GHRS, we will publish what we hope will be a definitive value for the $\mathrm{D} / \mathrm{H}$ ratio and interstellar temperature and turbulent velocity for this line of sight.

This work is supported by NASA Grant S-56500-D to the National Institute of Standards and Technology. I thank Alec Brown, Ken Gayley, and Blair Savage for their work on this project.

\section{References}

Ayres, T.R., Linsky, J.L. 1980, Ap. J. 241, 279.

Bruhweiler, F.C., Vidal-Madjar, A. 1987, in Exploring the Universe with the IUE Satellite, ed. Y. Kondo (Dordrecht: Reidel), p. 467.

Murthy, J., Henry, R.C., Moos, H.W., Vidal-Madjar, A., Linsky, J.L., Gry, C. 1990, Ap. J. 356, 223. 


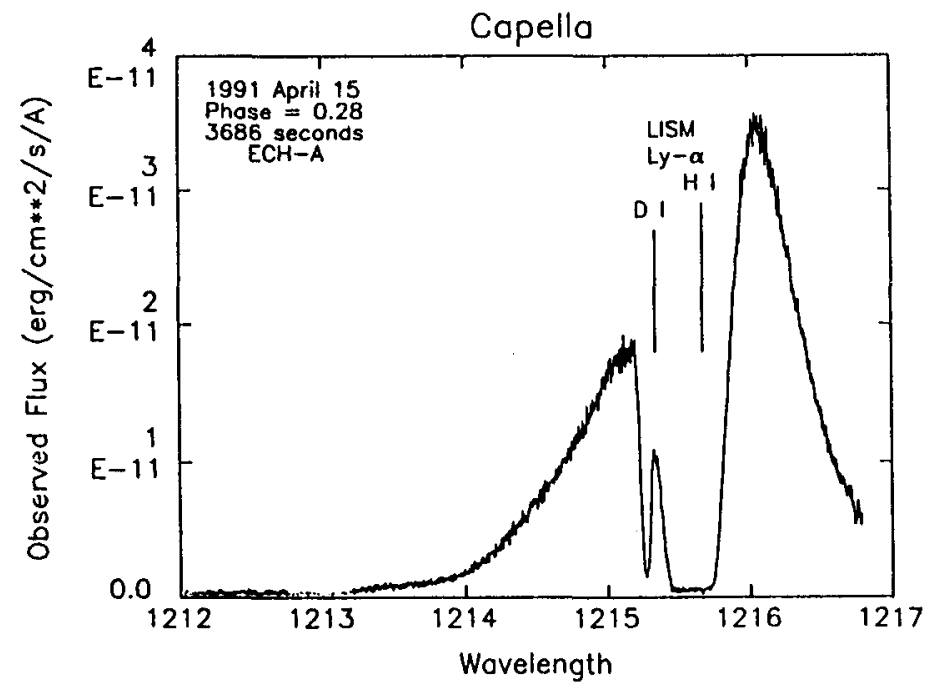

Figure 1: A GHRS high-dispersion spectrum of Capella obtained with the Ech-A grating. Superimposed on the stellar Lyman- $\alpha$ emission line is interstellar absorption due to hydrogen and deuterium.

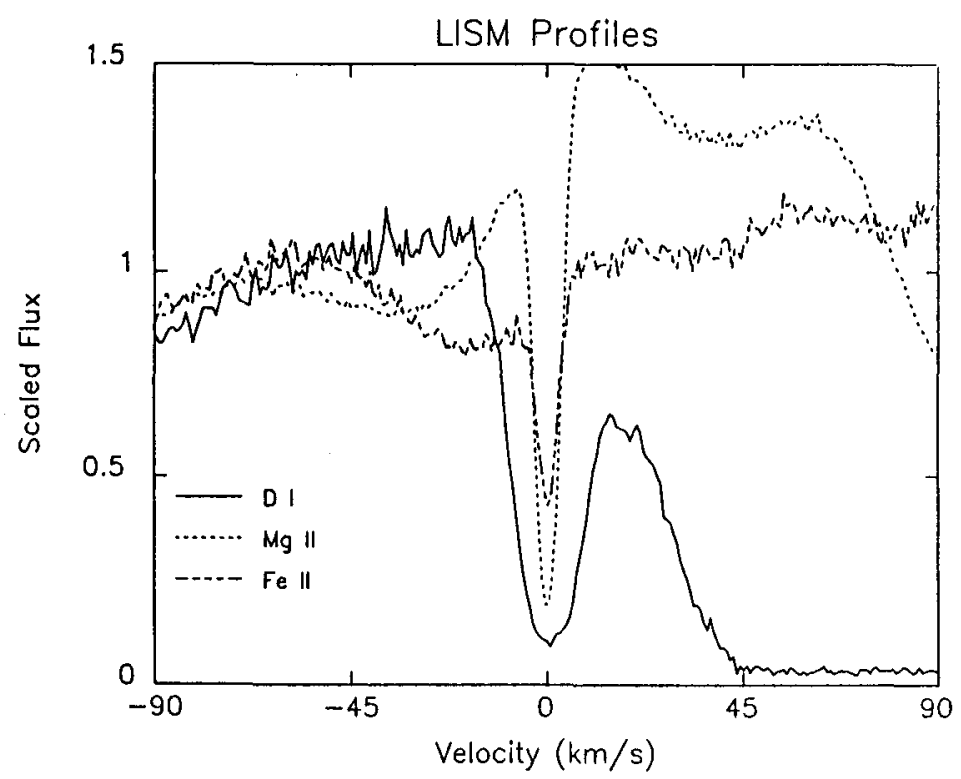

Figure 2: Comparison of the interstellar absorption lines of D I, Mg II, and Fe II on a common velocity scale. 